\title{
STUDY OF COMMUNICATION SKILLS IN CHILDREN WITH ATTENTION DEFICIT/ HYPERACTIVITY DISORDER IN SHAHREKORD
}

\author{
Habib Shahabi* \\ Payam Noor University \\ of Tehran, Iran
}

\author{
Dr. Maryamsadat \\ Ghiasian** \\ Payam Noor University \\ of Tehran, Iran
}

\author{
Dr. Mohammadreza \\ Ahmadkhani*** \\ Payam Noor University \\ of Tehran, Iran
}

\section{ABSTRACT}

Objective: This study was to investigate those pragmatic skills that children with attention deficit/hyperactivity disorder, especially children with pragmatic impairment, show in their colloquial interactions with their parents.

Method: In this regard, all children aged 6 to 13 years old with a diagnostic criterion for attention deficit/hyperactivity disorder in a a center for learning disabilities in Shahrekord were selected as research participants in a census method. Required data were collected by using an ADHD rating scale-IV (DuPaul et al., 1998) as well as a Five to fifteen parent questionnaire and were examined through descriptive statistics and chi square test methods.

Results: Findings showed that children with more hyperactivity were of more problem, especially in nonverbal skills $(\mathrm{r}=0.079 ; \mathrm{p} \leq 0.05)$ and subject Maitaining skills $(\mathrm{r}=0.032, \mathrm{p} \leq 0 / 05)$, than children with more attention deficit $(\mathrm{r}=0.00 ; \mathrm{p} \leq 0.05)$. In addition, compared to girls, boys had more problems in subject Maitaining skills $(\mathrm{p}=0.02)$ and non-verbal ones $(\mathrm{p}=0.014)$. The results of the analysis also showed that most issues related to pragmatic skills occur at younger ages and decrease with age. The study of previous research literature also confirmed that the low level of such skills can have adverse consequences for such children and pose serious risks to their future psychologically, educationally and behaviorally.

Conclusion: Psychological interventions and educational strategies seem necessary to increase the level of these skills in children with this disorder.

Keywords: Attention deficit/hyperactivity disorder, pragmatic skills, Language impairment, communication skills

\footnotetext{
* M.A. Graduate of Linguistics, University of Tehran-Jonoob Payame Noor, Tehran, Iran

** Associate Professor, Department of Language and Linguistics, University of Tehran-Jonoob Payame Noor, Tehran, Iran

*** Associate Professor, Department of Language and Linguistics, University of Tehran-Jonoob Payame Noor, Tehran, Iran
} 


\section{INTRODUCTION}

Attention deficit hyperactive disorder (ADHD) is one of the most common neurodevelopmental conditions in children (Shahim, Mehrangiz\& Yousefi, 2005); where according to American Psychiatric Association, it approximately includes $3-7 \%$ of all children who have diagnostic criteria for attention deficit/ hyperactivity disorder. As well, based on the Diagnostic Manual for Mental Disorders (DSM-IV fourth edition), this percentage is estimated at 5 to $5.8 \%$ by a group of Iranian scholars (Abdollahian, Shakeri \& Vosough, 2004). These children have experienced some levels of hyperactivity, impulsive behavior and attention deficit that significantly affects their performance in their various areas of daily life at home, school, and in other social interactions.

The results of research indicate that although most people with ADHD are diagnosed with their illness in childhood, its impacts continue to increase as they age in order to endanger their social status. Some of the long- term negative effects of this disorder, found in various studies are: low educational achievement, job instability, the risk of antisocial or criminal behavior, higher rates of adolescent pregnancy and increased risk of personality trauma and other psychiatric diagnosis (Barkly, 2002; Biederman et al., 1996; Fisherman et al., 2002; Weis and Hechtman, 1993; Whalen and Henker, 1991). Overall, these findings implicitly indicate the existence of a negative effect on the social development of people with attention deficit/hyperactivity disorder in adulthood.

Research prioritizes communicative competence among other factors involved in the formation of a suitable social status (Eliss, 1999; Gallagher, 1991; Mc Tear and Conti-Ramsden, 1992; Winsler, Diaz, Mc Carty, Atencio, and Chabay, 2000). Indeed, the ability to use language to communicate (i.e., knowledge of language use) is 'an intrinsic social phenomenon' (Gallagher, 2000). The unresolved interdependence of skills and social development provides a condition in which the role of ADHD is considered as a factor, reducing the social character of affected people. For example, in his review of the outcomes of life with this disorder, Barkly noted that over $54 \%$ of children experience the attention/hyperactivity disorder with speech problems, related to their implementation process. He has also identified an increase in the incidence of speech complications as well as issues related to the language of use associated with this disorder (Barkly, 2002, p18). While, the study does not endorse Baird, Stevenson, and Williamson radical proposal (2000), based on which the attention deficit/hyperactivity disorder could be more accurately perceived as a communicative disorder, it still considers the need for research to clarify the relationship between attention deficit/ hyperactivity disorder and linguistic problems especially pragmatic ones.

Moreover, Staikova et al. (2013) mentioned the existence of social problems in $52 \%$ to $82 \%$ of children with attention deficit/hyperactivity disorder and cited it as one of the important features of this disorder. Referring to the 
findings of some researchers (e.g., Barclay, 1997; Hagens et al., 2000; Mikami et al., 2011), they has been noted that the social issues related with the disorder were primarily linked with impulsive behavior (problems such as interruption of conversation, inability to wait for the turn) and then to attention deficits (problems such as poor listening). Also, considering that communication requires the ability to start a conversation, respond, and maintain attention, Bruce et al. (2006) have stated that it is not surprising to say that there are different symptoms of the disorder are associated with language functional deficits.

Collective view has suggested the current comorbidity of attention deficit/ hyperactivity disorder impacts with the individual social development, and that over $50 \%$ of children with an inattention hyperactivity diagnosis have a linguistic impairment. In linguistic studies, all the studies related to linguistic disorder effect on the individuals' socialization process have focused on communicative competence, (Adams \& Bishop, 1989; Bishop et al, 2000; Cohn, Davine, Horodezky, Lipsset, \& Isaacson, 1993; Gallagher, 1991; Turkstra, Mc Donald, \& Kaufmann, 1995; Prutting, 1982).

On the other hand, few studies in the world have focused on topics specifically addressing the symptoms of attention deficit/hyperactivity disorder symptoms and pragmatic skills. In a Collaborative Study of ADHD, Tannock and Brown (2000) reviewed the exploratory research on the relationship between attention deficit/ hyperactivity disorder and pragmatic problems and identified nine studies that has been investigating specific pragmatic impairments in children with ADHD. Most of the studies sampled children between the ages of 6 and 12 (though, studies related to adult groups can be summarized in two cases: Friedman et al.; von Brysen, 2007). Meanwhile, in our country, Iran, there does not appear to be any similar studies that address the specific pragmatic impairment of children (or even adults) with ADHD. Of course, the lack of research on this subject does not reduce the credibility of extensive empirical data collected from thousands of affected children or parents, teachers, and clinicians who detected ADHD, or interacted with or taken care of children with it. However, this indicates the need for the present study to investigate the relationship between ADHD and the potential impacts that this disorder has on the pragmatic aspects of communicative competence. In fact, understanding the effects of attention deficit/hyperactivity disorder is the first step in addressing the negative consequences of this disorder.

Due to the $5.8 \%$ prevalence of ADHD in the Iranian child community (statistics reported in the work of Abdullahian and his colleagues) on the one hand, and the continuation of long-term effects of the disorder as children age raise on the other hand, it seems necessary to examine the effects of ADHD central symptoms as well as the effects of age and gender on the pragmatic aspects of these children's communicative competence. This will help the related experts to achieve an appropriate intervention solution to prevent 
the development and improvement of comprehension and communication problems in children with ADHD.

Moreover, given that communication requires the initiation, response, and maintenance of attention, it is not surprising to note that multiple symptoms of ADHD are associated with pragmatic (language use) deficits (Bruce, Ternvand, Netlebladt 2006). In this regard, Kamarata and Gibson (1999) examined ADHD's symptoms in relation with language skills and showed that both signs of inattention and hyperactivity/impulsivity can interfere in the verbal/ non-verbal aspects of communication, including maintaining subject and maintaining appropriate physical proximity. Also, communicative deficits in the language of children with ADHD have been reported by both parents and teachers (Bingel and Cain, 2007; Bishop and Baird, 2001; Humphreys, Colton, Malone, and Roberts, 1994). Bishop and Baird (2001) also found that children with ADHD not only had more communicative deficits and social problems than normal children, but also their language proficiency levels were different in only a few cases from children with autism. As with autism, the problems of children with attention deficit/hyperactivity disorder regarding language use have been relatively greater than their problems in relation to the language structure (Gorts and Ambrecht, 2008; Gorts et al., 2004).

While pragmatic deficits have been linked to social disorders in other clinical communities, to date almost no studies in our country have examined the existence of a similar association with attention deficit/hyperactivity disorder. Therefore, it was felt necessary to examine those colloquial skills or communicative deficits that children with diagnostic criteria for this disorder show in their colloquial interactions with their parent. According to this goal, the main assumption tested in this study is as follows: "there is a significant relationship between children with ADHD and the effect of the four mediating factors (i.e. hyperactivity, attention deficit, age and gender) and pragmatic problems (including the problems of non-verbal communication, verbal communication, subject maintenance and basic social language skills)'.

Rest of the study is organized as follows: in the next part, research methodology and statistical analyses are presented. After that, we will provide the results of the study. Then, we will discuss about the results. Finally, we conclude the study.

\section{METHODOLOGY}

\subsection{PARTICIPANTS}

The sample group was equal to the statistical population, including all students (16 boys and 18 girls, with an average age of 9 years) with a diagnosis of Attention Deficit/Hyperactivity Disorder, which in the academic year of 2015-16 in the Center for Learning Disabilities Related to Education District 2, Shahrekord (Chaharmahal and Bakhtiari province capital) had enrolled. Majority of these 
children had not previously received any therapeutic intervention for their linguistic problem from a speech/language pathologist. Census sampling was done here.

\subsection{PROCEDURES}

Children and their parents referred to the Center for Learning Disabilities and underwent a 1-hour assessment during which several tests performed to assess the level of their performance in verbal language and pragmatic skills. Following this assessment, the assessor also provided the parent a Deficit/ Hyperactivity Disorder Questionnaire. All measurements in this questionnaire were as follows:

Diagnostic Assessments: In this regard, the fourth version of the Attention Deficit/Hyperactivity Disorder Questionnaire (DuPaul, Power, Anastopoulos; Ride, 1998) was used, while some items had been omitted. In general, the questionnaire consisted of three parts: non-verbal skills part (Skills of sending and receiving messages and communicating via non-verbal messages or through body gestures; 5 items), verbal skills part (including Speaking

Skills, 3 items), and general colloquial skills (effective communication skills involving all aspects of effective attention/listening with body,verbal communication (speaking), non-verbal communication (tone, postures and movements), trust, empathy, and talking; 17 items).

Assessment of pragmatic skills: In order to assess the children performance in pragmatic skills, their parents were asked to complete a Five to Fifteen Parental Questionnaire. This questionnaire is partly adapted from standard questionnaire of 5 to 15 (Kadsjo et al., 2004).

Statistical analysis: Data analysis was performed using Social Science using Statistical Packages (SPSS) software 18.0. Research materials were described using frequencies, percentages, means, medians, and 90th percentiles. Correlation were obtained through Pearson product instantaneous correlations test. T-tests were also used to examine the differences between groups. Factor analysis of the questionnaire was performed by principal component analysis with all varimax rotation degree, and an alpha level of $0.05 \%$ was considered for statistical tests.

\section{RESULTS}

\subsection{RESULTS FROM INFERENTIAL STATISTICS}

Table 1 shows the results of the Pearson correlation analysis test, which is based on the main hypothesis of the research. According to the data in this table, the results of inferential research statistics are:

(Hyperactivity factor of) ADHD has a negative and significant effect on the aspects of non-verbal communication as a factor of communicative 


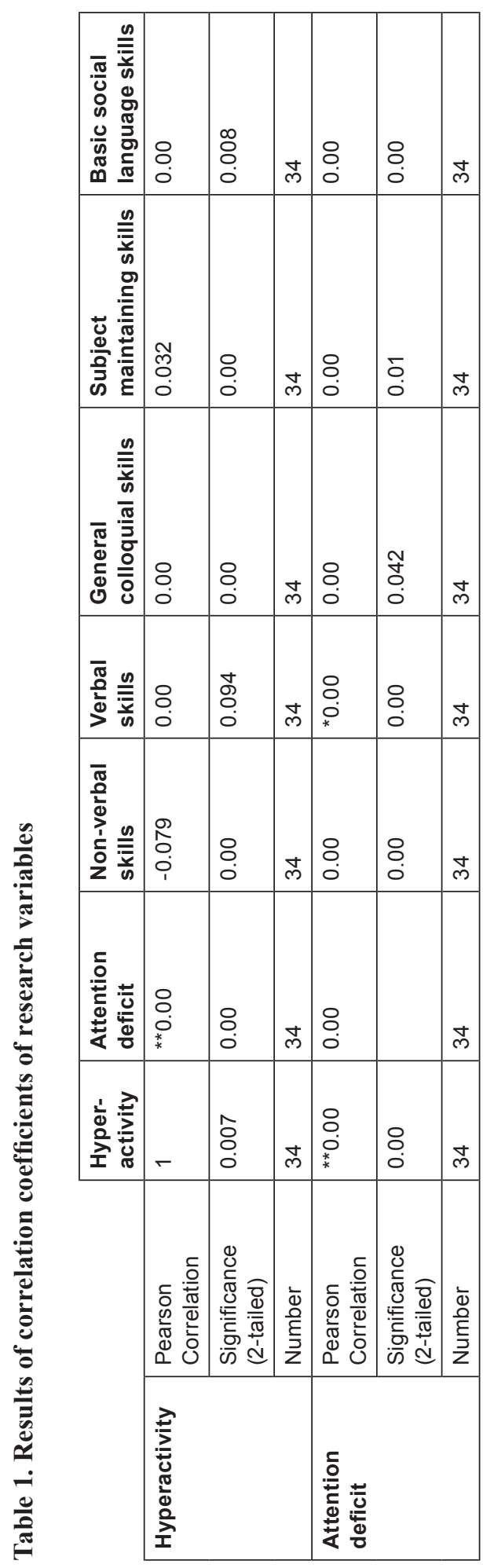


competence $(p=0 / 079)$. This is while attention deficit factor of ADHD had a positive and significant effect on the aspects of non- verbal communication as a factors of communicative competence $(p=0 / 064)$.

(Hyperactivity factor of) ADHD had a negative and significant effect on the aspect of verbal communication as a factor of communicative competence $(\mathrm{p}=0 / 094)$.

(Hyperactivity factor of) ADHD had a positive and significant effect on the aspect of general colloquial skills $(p \leq 0 / 05)$, subject maintaining $(p=0 / 032)$, and basic social language skills $(\mathrm{p} \leq 0 / 01)$ as the other factors of communicative competence.

\subsection{SUB-CATEGORIES RELATED TO PRAGMATIC LANGUAGE}

The three sub-categories belonging to pragmatic language are organized as follows: a) non-verbal skills (5 items; median2); b) verbal skills (3 items; median1); and c) general colloquial skills (15items, median2), which is in turn divided into two further sub- categories, namely "subject maintaining" (6 items; median2) and "basic social language" ( 3 items; median1).

To further compare the relationship among pragmatic skills' subcategories, cross-tables were provided in which sub-categories were in turn divided into three minor sub-categories: No/a little (No, $<1)$, and average to severe (mean $\geq 1)$.

According to table 2, 26 subjects had severe problems in non-verbal skills, of which 7 were without hyperactivity and 3 without attention deficit. There were a group of 10 of children who had both hyperactivity and attention deficit problems. Also, there was a group of 3 children with severe problems in nonverbal skills, who had not attention deficit, and also there was a group of 7 children without hyperactivity problems (see table 2 ).

Table 2. The relationship between non-verbal skills and attention deficit/ hyperactivity

\begin{tabular}{|c|c|c|c|c|c|c|c|c|c|c|}
\hline \multicolumn{2}{|l|}{ GT } & \multicolumn{4}{|c|}{ Attention deficit } & \multicolumn{5}{|c|}{ Hyperactivity } \\
\hline $\begin{array}{c}\text { GT } \\
=\end{array}$ & & Never & $\begin{array}{l}\text { Some } \\
\text { times }\end{array}$ & Often & $\begin{array}{l}\text { Most } \\
\text { often }\end{array}$ & Total & Never & $\begin{array}{l}\text { Some } \\
\text { times }\end{array}$ & Often & Total \\
\hline \multirow{3}{*}{ 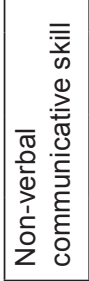 } & Disa & $\begin{array}{l}1 \\
100 \%\end{array}$ & $\begin{array}{l}0 \\
0 / 0 \%\end{array}$ & $\begin{array}{l}0 \\
0 / 0 \% \\
\end{array}$ & \begin{tabular}{|l|}
0 \\
$0 / 0 \%$ \\
\end{tabular} & $\begin{array}{l}1 \\
100 \% \\
\end{array}$ & $\begin{array}{l}0 \\
0 / 0 \% \\
\end{array}$ & $\begin{array}{l}1 \\
100 \% \\
\end{array}$ & $\begin{array}{l}0 \\
0 / 0 \%\end{array}$ & $\begin{array}{l}1 \\
100 \% \\
\end{array}$ \\
\hline & $\begin{array}{l}\text { Sometimes/ } \\
\text { somehow }\end{array}$ & $\begin{array}{l}1 \\
114 \%\end{array}$ & $\begin{array}{l}1 \\
14 \%\end{array}$ & $\begin{array}{l}3 \\
42 \%\end{array}$ & $\begin{array}{l}2 \\
28 \%\end{array}$ & $\begin{array}{l}7 \\
100 \%\end{array}$ & $\begin{array}{l}4 \\
57 \%\end{array}$ & $\begin{array}{l}2 \\
28 \%\end{array}$ & $\begin{array}{l}1 \\
14 \%\end{array}$ & $\begin{array}{l}7 \\
100 \%\end{array}$ \\
\hline & Agre & $\begin{array}{l}3 \\
11 \% \\
\end{array}$ & \begin{tabular}{|l|}
11 \\
$42 \%$ \\
\end{tabular} & $\begin{array}{l}10 \\
38 \% \\
\end{array}$ & \begin{tabular}{|l|l|}
2 \\
$7 \%$ \\
\end{tabular} & $\begin{array}{l}26 \\
100 \% \\
\end{array}$ & $\begin{array}{l}7 \\
32 \% \\
\end{array}$ & \begin{tabular}{|l}
9 \\
$35 \%$ \\
\end{tabular} & $\begin{array}{l}10 \\
32 \% \\
\end{array}$ & $\begin{array}{l}26 \\
100 \% \\
\end{array}$ \\
\hline \multicolumn{2}{|l|}{ Total } & $\begin{array}{l}5 \\
14 \%\end{array}$ & $\begin{array}{l}12 \\
35 \%\end{array}$ & $\begin{array}{l}13 \\
38 \%\end{array}$ & $\begin{array}{l}4 \\
11 \%\end{array}$ & $\begin{array}{l}34 \\
100 \%\end{array}$ & $\begin{array}{l}11 \\
32 \%\end{array}$ & $\begin{array}{l}12 \\
35 \%\end{array}$ & $\begin{array}{l}11 \\
32 \%\end{array}$ & $\begin{array}{l}34 \\
100 \%\end{array}$ \\
\hline
\end{tabular}


202012

According table 3, there was a group of 14 children with severe verbal communication problems, where 3 children had severe problems in verbal communication and serious problems of hyperactivity. There was also a group of 1 children only with serious problems in hyperactivity without serious problems in verbal skill (see table 3).

Table 3. The relationship between verbal communicative skills and attention deficit/hyperactivity

\begin{tabular}{|c|c|c|c|c|c|c|c|c|c|c|}
\hline & \multicolumn{5}{|c|}{ Attention deficit } & \multicolumn{4}{|c|}{ Hyperactivity } \\
\hline & & Never & $\begin{array}{l}\text { Some } \\
\text { times }\end{array}$ & Often & $\begin{array}{l}\text { Most } \\
\text { often }\end{array}$ & Total & Never & $\begin{array}{l}\text { Some } \\
\text { times }\end{array}$ & Often & Total \\
\hline \multirow{3}{*}{ 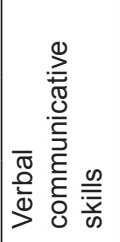 } & Disagree & $\begin{array}{l}0 \\
0 / 0 \%\end{array}$ & \begin{tabular}{|l|}
0 \\
$0 / 0 \%$ \\
\end{tabular} & $\begin{array}{l}2 \\
66 \% \\
\end{array}$ & $\begin{array}{l}1 \\
33 \% \\
\end{array}$ & \begin{tabular}{|l|}
3 \\
$100 \%$ \\
\end{tabular} & $\begin{array}{l}0 \\
0 / 0 \%\end{array}$ & \begin{tabular}{|l|}
1 \\
$33 \%$ \\
\end{tabular} & $\begin{array}{l}2 \\
66 \% \\
\end{array}$ & $\begin{array}{l}3 \\
100 \% \\
\end{array}$ \\
\hline & $\begin{array}{l}\text { Sometimes/ } \\
\text { somehow }\end{array}$ & $\begin{array}{l}2 \\
11 \% \\
\end{array}$ & \begin{tabular}{|l|}
6 \\
$35 \%$
\end{tabular} & $\begin{array}{l}6 \\
35 \% \\
\end{array}$ & \begin{tabular}{|l}
3 \\
$17 \%$ \\
\end{tabular} & $\begin{array}{l}17 \\
100 \%\end{array}$ & $\begin{array}{l}5 \\
29 \%\end{array}$ & \begin{tabular}{|l}
5 \\
$29 \%$ \\
\end{tabular} & $\begin{array}{l}7 \\
41 \%\end{array}$ & $\begin{array}{l}17 \\
100 \%\end{array}$ \\
\hline & Agree & \begin{tabular}{|l|}
3 \\
$21 \%$ \\
\end{tabular} & \begin{tabular}{|l|}
6 \\
$42 \%$ \\
\end{tabular} & $\begin{array}{l}5 \\
35 \% \\
\end{array}$ & \begin{tabular}{|l|l}
0 \\
$0 / 0 \%$ \\
\end{tabular} & \begin{tabular}{|l|}
14 \\
$100 \%$ \\
\end{tabular} & $\begin{array}{l}6 \\
42 \% \\
\end{array}$ & \begin{tabular}{|l|}
6 \\
$42 \%$ \\
\end{tabular} & \begin{tabular}{|l|}
2 \\
$14 \%$ \\
\end{tabular} & $\begin{array}{l}14 \\
100 \% \\
\end{array}$ \\
\hline \multicolumn{2}{|l|}{ Total } & \begin{tabular}{|l|}
5 \\
$14 \%$
\end{tabular} & $\begin{array}{l}12 \\
35 \%\end{array}$ & $\begin{array}{l}13 \\
38 \%\end{array}$ & \begin{tabular}{|l|}
4 \\
$11 \%$
\end{tabular} & $\begin{array}{l}34 \\
100 \%\end{array}$ & \begin{tabular}{|l|}
11 \\
$32 \%$
\end{tabular} & \begin{tabular}{|l|}
12 \\
$32 \%$
\end{tabular} & \begin{tabular}{|l|}
11 \\
$35 \%$
\end{tabular} & $\begin{array}{l}34 \\
100 \%\end{array}$ \\
\hline
\end{tabular}

According to table 4, a group of 15 children with severe problems in their general colloquial skills were observed, of which 7 children had severe problems with non-verbal and their general colloquial skills and without hyperactivity. Here, a group of 3 children had severe problems with general colloquial skills, of which one third (1 child) had attention deficit. There was also a group of 8 children, all of which had moderate degree of problem in their general colloquial skills without attention deficit. A group of 10 children was also observed that most of them had attention deficit and hyperactivity problems with average degree of problems in their general colloquial skills (see table 4).

Table 4. The relationship between general colloquial skills and attention deficit/ hyperactivity

\begin{tabular}{|c|c|c|c|c|c|c|c|c|c|c|}
\hline & \multicolumn{5}{|c|}{ Attention deficit } & \multicolumn{4}{|c|}{ Hyperactivity } \\
\hline & & Never & $\begin{array}{l}\text { Some } \\
\text { times }\end{array}$ & Often & $\begin{array}{l}\text { Most } \\
\text { often }\end{array}$ & Total & Never & $\begin{array}{l}\text { Some } \\
\text { times }\end{array}$ & Often & Total \\
\hline \multirow{3}{*}{ 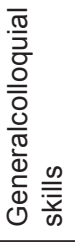 } & Disagree & $\begin{array}{l}1 \\
50 \%\end{array}$ & $\begin{array}{l}0 \\
0 / 0 \%\end{array}$ & $\begin{array}{l}0 \\
0 / 0 \%\end{array}$ & $\begin{array}{l}1 \\
50 \%\end{array}$ & $\begin{array}{l}2 \\
100 \%\end{array}$ & $\begin{array}{l}0 \\
0 / 0 \%\end{array}$ & $\begin{array}{l}2 \\
100 \%\end{array}$ & $00 / 0 \%$ & $\begin{array}{l}2 \\
100 \%\end{array}$ \\
\hline & $\begin{array}{l}\text { Sometimes/ } \\
\text { somehow }\end{array}$ & $\begin{array}{l}1 \\
5 \%\end{array}$ & $\begin{array}{l}4 \\
23 \%\end{array}$ & $\begin{array}{l}10 \\
58 \%\end{array}$ & $\begin{array}{l}2 \\
11 \%\end{array}$ & $\begin{array}{l}17 \\
100 \%\end{array}$ & $\begin{array}{l}4 \\
23 \%\end{array}$ & $\begin{array}{l}3 \\
17 \%\end{array}$ & $\begin{array}{l}10 \\
58 \%\end{array}$ & $\begin{array}{l}17 \\
100 \%\end{array}$ \\
\hline & Agree & $\begin{array}{l}3 \\
20 \%\end{array}$ & $\begin{array}{l}8 \\
53 \% \\
\end{array}$ & $\begin{array}{l}3 \\
20 \% \\
\end{array}$ & $\begin{array}{l}1 \\
6 \%\end{array}$ & $\begin{array}{l}15 \\
100 \%\end{array}$ & $\begin{array}{l}7 \\
46 \%\end{array}$ & $\begin{array}{l}7 \\
46 \% \\
\end{array}$ & $\begin{array}{l}1 \\
6 \%\end{array}$ & $\begin{array}{l}15 \\
100 \% \\
\end{array}$ \\
\hline \multicolumn{2}{|l|}{ Total } & $\begin{array}{l}5 \\
14 \%\end{array}$ & $\begin{array}{l}12 \\
35 \%\end{array}$ & $\begin{array}{l}13 \\
38 \%\end{array}$ & $\begin{array}{l}4 \\
11 \%\end{array}$ & $\begin{array}{l}34 \\
100 \%\end{array}$ & $\begin{array}{l}11 \\
32 \%\end{array}$ & $\begin{array}{l}12 \\
35 \%\end{array}$ & $\begin{array}{l}11 \\
32 \%\end{array}$ & $\begin{array}{l}34 \\
100 \%\end{array}$ \\
\hline
\end{tabular}


According to table 5, a group of 19 children had serious problems in their "subject maintaining" skills, of which one third were without attention deficit, and there was a group of 6 who had serious problems in their subject maintaining skills, with only one in six having hyperactivity symptoms. There was also a 1-person group that had serious problems of attention deficit, without subject maintaining complications (see table 5).

Table 5. The relationship between subject maintaining skills and attention deficit/ hyperactivity

\begin{tabular}{|c|c|c|c|c|c|c|c|c|c|c|}
\hline & \multicolumn{5}{|c|}{ Attention deficit } & \multicolumn{4}{|c|}{ Hyperactivity } \\
\hline & & Never & \begin{tabular}{|l} 
Some \\
times
\end{tabular} & Often & $\begin{array}{l}\text { Most } \\
\text { often }\end{array}$ & Total & Never & $\begin{array}{l}\text { Some } \\
\text { times }\end{array}$ & Often & Total \\
\hline \multirow{3}{*}{ 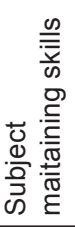 } & Disagree & $\begin{array}{l}2 \\
40 \% \\
\end{array}$ & \begin{tabular}{|l|l}
0 \\
$0 / 0 \%$ \\
\end{tabular} & $\begin{array}{l}2 \\
40 \% \\
\end{array}$ & $\begin{array}{l}1 \\
20 \% \\
\end{array}$ & $\begin{array}{l}5 \\
100 \% \\
\end{array}$ & $\begin{array}{l}2 \\
40 \% \\
\end{array}$ & $\begin{array}{l}2 \\
40 \% \\
\end{array}$ & $\begin{array}{l}1 \\
20 \% \\
\end{array}$ & $\begin{array}{l}5 \\
100 \% \\
\end{array}$ \\
\hline & $\begin{array}{l}\text { Sometimes/ } \\
\text { somehow }\end{array}$ & \begin{tabular}{|l|l|}
0 \\
$0 / 0 \%$ \\
\end{tabular} & \begin{tabular}{|l|}
3 \\
$30 \%$ \\
\end{tabular} & $\begin{array}{l}4 \\
40 \% \\
\end{array}$ & $\begin{array}{l}3 \\
30 \% \\
\end{array}$ & $\begin{array}{l}10 \\
100 \% \\
\end{array}$ & $\begin{array}{l}3 \\
30 \% \\
\end{array}$ & $\begin{array}{l}3 \\
30 \% \\
\end{array}$ & $\begin{array}{l}4 \\
40 \% \\
\end{array}$ & $\begin{array}{l}10 \\
100 \% \\
\end{array}$ \\
\hline & Agree & \begin{tabular}{|l|}
3 \\
$15 \%$
\end{tabular} & $\begin{array}{l}9 \\
47 \%\end{array}$ & $\begin{array}{l}7 \\
36 \%\end{array}$ & $\begin{array}{l}0 \\
0 / 0 \%\end{array}$ & $\begin{array}{l}19 \\
100 \%\end{array}$ & $\begin{array}{l}6 \\
31 \%\end{array}$ & $\begin{array}{l}7 \\
36 \%\end{array}$ & $\begin{array}{l}6 \\
31 \%\end{array}$ & $\begin{array}{l}19 \\
100 \%\end{array}$ \\
\hline \multicolumn{2}{|l|}{ Total } & \begin{tabular}{|l|}
5 \\
$14 \%$
\end{tabular} & $\begin{array}{l}12 \\
35 \%\end{array}$ & $\begin{array}{l}13 \\
38 \%\end{array}$ & $\begin{array}{l}4 \\
11 \%\end{array}$ & $\begin{array}{l}34 \\
100 \%\end{array}$ & $\begin{array}{l}11 \\
32 \%\end{array}$ & $\begin{array}{l}12 \\
35 \%\end{array}$ & $\begin{array}{l}11 \\
32 \%\end{array}$ & $\begin{array}{l}34 \\
100 \%\end{array}$ \\
\hline
\end{tabular}

According to table 6, a group of 11 children had serious problems in their "basic social language" skills, of which one child with serious problem in basic social language skills was without attention deficit. There were 4-children who had serious problems in basic social language skills without any hyperactivity symptoms. There was also a 19-children group having moderate problems in basic social language skills, which among them 7-ones had problems in their basic social language skills without any hyperactivity symptoms. There were also 6-children all with moderate degree of problems in basic social language skills, this was while two of them had often inattention deficit and 3 of them had often hyperactivity symptoms.

Table 6. The relationship between basic social language skills and attention deficit/hyperactivity

\begin{tabular}{|c|c|c|c|c|c|c|c|c|c|c|}
\hline & \multicolumn{5}{|c|}{ Attention deficit } & \multicolumn{4}{|c|}{ Hyperactivity } \\
\hline & & Never & $\begin{array}{l}\text { Some } \\
\text { times }\end{array}$ & Often & $\begin{array}{l}\text { Most } \\
\text { often }\end{array}$ & Total & Never & $\begin{array}{l}\text { Some } \\
\text { times }\end{array}$ & Often & Total \\
\hline \multirow{3}{*}{ 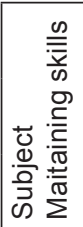 } & Dis & \begin{tabular}{|l|}
2 \\
$40 \%$ \\
\end{tabular} & \begin{tabular}{|l|}
0 \\
$0 / 0 \%$ \\
\end{tabular} & $\begin{array}{l}2 \\
40 \% \\
\end{array}$ & \begin{tabular}{|l|}
1 \\
$20 \%$ \\
\end{tabular} & $\begin{array}{l}5 \\
100 \% \\
\end{array}$ & \begin{tabular}{|l|}
2 \\
$40 \%$ \\
\end{tabular} & \begin{tabular}{|l|}
2 \\
$40 \%$ \\
\end{tabular} & $\begin{array}{l}1 \\
20 \% \\
\end{array}$ & $\begin{array}{l}5 \\
100 \% \\
\end{array}$ \\
\hline & \begin{tabular}{|l}
$\begin{array}{l}\text { Sometimes/ } \\
\text { somehow }\end{array}$ \\
\end{tabular} & \begin{tabular}{|l|l|}
0 \\
$0 / 0 \%$ \\
\end{tabular} & \begin{tabular}{|l|}
3 \\
$30 \%$ \\
\end{tabular} & \begin{tabular}{|l|}
4 \\
$40 \%$ \\
\end{tabular} & \begin{tabular}{|l|}
3 \\
$30 \%$ \\
\end{tabular} & $\begin{array}{l}10 \\
100 \% \\
\end{array}$ & \begin{tabular}{|l|}
3 \\
$30 \%$ \\
\end{tabular} & \begin{tabular}{|l|}
3 \\
$30 \%$ \\
\end{tabular} & $\begin{array}{l}4 \\
40 \% \\
\end{array}$ & $\begin{array}{l}10 \\
100 \% \\
\end{array}$ \\
\hline & Agree & \begin{tabular}{|l|}
3 \\
$15 \%$ \\
\end{tabular} & \begin{tabular}{|l|}
9 \\
$47 \%$ \\
\end{tabular} & \begin{tabular}{|l|}
7 \\
$36 \%$ \\
\end{tabular} & \begin{tabular}{|l|l|}
0 \\
$0 / 0 \%$ \\
\end{tabular} & $\begin{array}{l}19 \\
100 \%\end{array}$ & \begin{tabular}{|l|}
6 \\
$31 \%$ \\
\end{tabular} & \begin{tabular}{|l|}
7 \\
$36 \%$ \\
\end{tabular} & $\begin{array}{l}6 \\
31 \%\end{array}$ & $\begin{array}{l}19 \\
100 \% \\
\end{array}$ \\
\hline \multicolumn{2}{|l|}{ Total } & $\begin{array}{l}5 \\
14 \%\end{array}$ & \begin{tabular}{|l|}
12 \\
$35 \%$
\end{tabular} & $\begin{array}{l}13 \\
38 \%\end{array}$ & \begin{tabular}{|l|}
4 \\
$11 \%$
\end{tabular} & $\begin{array}{l}34 \\
100 \%\end{array}$ & \begin{tabular}{|l|}
11 \\
$32 \%$
\end{tabular} & \begin{tabular}{|l|}
12 \\
$35 \%$
\end{tabular} & $\begin{array}{l}11 \\
32 \%\end{array}$ & $\begin{array}{l}34 \\
100 \%\end{array}$ \\
\hline
\end{tabular}




\subsection{FACTOR ANALYSIS OF THE QUESTIONNAIRE SUB-DOMAINS}

Table 7 shows the results of present study with an Eigen value $>1$. According to this table, those five factors that reflect the distinctly clinically sensitive areas have been able to justify between $23 \%$ and $85 \%$ of the total variation. The following six factors along with their loading on the questionnaire reveal the main area of attention deficit hyperactivity disorder (ADHD). Percentage of children with a mean score of $1 \leq$ are shown below:

1) Non-verbal skills: eye contact, understanding communicative gestures, maintaining distance and closeness (appropriate) when communicating, noticing changes in facial expressions and noticing changes in tone of voice (39-76\% of total changes),

2) Verbal skills: ability to talk about experiences and situations in an understandable to the listener, to take turns during the conversation, to participate appropriately in a conversation (51-85\% of total changes),

3) General colloquial skills: interrupt the speech at the right time and place, repeat again when the message is not understood, give effective instructions to others, give enough information for the listener to understand, repeating when the message is not understood, asking the other person to speak more clearly or repeating the word, finding proper answers to questions, conversational maintaining skills, basic social language skills, articulating your wants, needs and preferences, asking for help appropriately, sharing your feelings appropriately with others, recognize words related to thanks and encouragement and use them appropriately, start and continue friendships, give appropriate explanations for activities, properly understand jokes and use them, and show your mood to convenient shape. (23-73\% of total changes),

And among general colloquial skills:

1) Subject maintaining: ability to start a conversation, enter the discussion properly, continue the topic, change the topic, close the topic, point out clues and tips, (52-74\% of total changes),

2) Basic social language skills: saying hello or goodbye, greetings, use polite terms; applying introductions (data not shown, because only one component was extracted and it was not possible to rotate the solution).

Accordingly, the highest explaining power belonged to the verbal skills factor (51-85\% of total changes), and then the general colloquial skills and non- verbal skills were respectively (39-76\% and $23-73 \%$ of total changes). 
Table 7. Rotated component matrix of sub-domains of the questionnaire based on Eigen value $>1$ and loading $>0 / 300$. Extraction method: principal component analysis. Rotation method: varimax with Kaiser Normalization

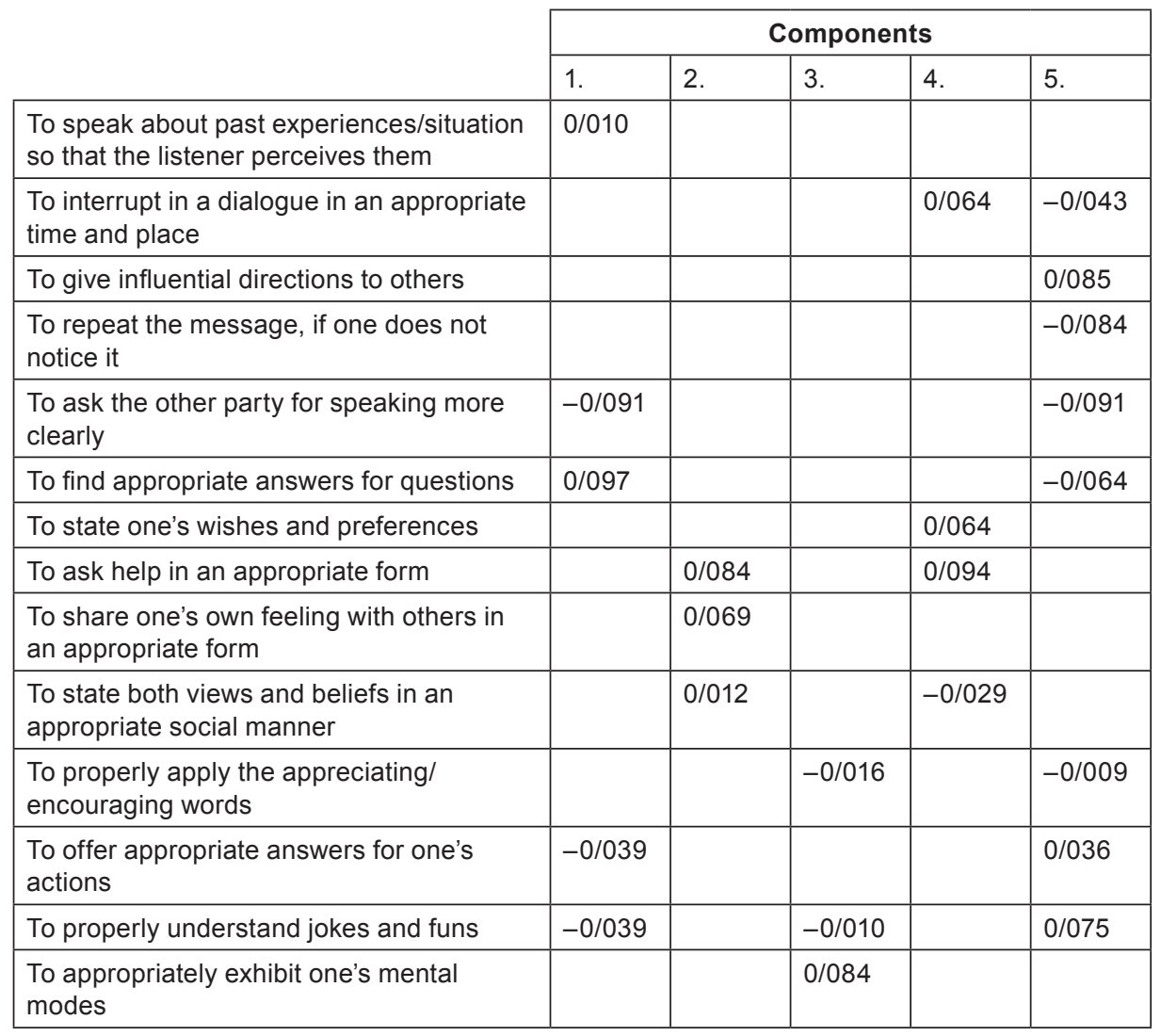

\subsection{ASSOCIATIONS BETWEEN AGE, GENDER, AND COMMUNICATIVE COMPETENCE FACTORS (PRAGMATIC SKILLS)}

According to table 8, a significant difference was found between boys $(\mathrm{N}=18)$ and girls $(\mathrm{N}=16)$ for non-verbal communication (mean values 1,$1 ; \mathrm{F}(34)=5)$, verbal communication (mean values 1, 1: F $(34)=4$ ), and general colloquial skills (mean values1, 1: F $(34)=0$ ). Similar gender differences were also observed about "subject maintaining" factors (respectively with mean values1, $1 ; \mathrm{F}(34)=0 / 035)$, and "basic social language skills" (respectively with mean values 1,$1 ; \mathrm{F}(34)=5$ ) (data shown in table 8 ). 
202012

Table 8. The relationship between gender and communicative competence factors (pragmatic skills): for girls $(\mathrm{N}=18$; hyperactivity mean value $=0$, attention deficit mean value $=1$ )

\begin{tabular}{|c|c|c|c|c|c|c|c|}
\hline & & \multicolumn{6}{|c|}{ Test value $=0$} \\
\hline & & \multirow[t]{2}{*}{ t-score } & \multirow[t]{2}{*}{\begin{tabular}{|l}
$\begin{array}{l}\text { Freedom } \\
\text { degree }\end{array}$ \\
\end{tabular}} & \multirow{2}{*}{$\begin{array}{l}\text { Significance } \\
\text { rating } \\
\text { (two-tailed) }\end{array}$} & \multirow[t]{2}{*}{$\begin{array}{l}\text { Mean } \\
\text { difference }\end{array}$} & \multicolumn{2}{|c|}{$\begin{array}{l}95 \% \text { difference } \\
\text { confidence interval }\end{array}$} \\
\hline & & & & & & Lowest & Highest \\
\hline Hyperactivity & \begin{tabular}{|l|} 
girls \\
boys
\end{tabular} & $\begin{array}{l}4 \\
4\end{array}$ & $\begin{array}{l}17 \\
15 \\
\end{array}$ & $\begin{array}{l}0 / 00 \\
0.00\end{array}$ & $\begin{array}{l}0 / 00 \\
1.063\end{array}$ & $\begin{array}{l}1 / 00 \\
0.00\end{array}$ & $\begin{array}{l}0 / 00 \\
0.00\end{array}$ \\
\hline $\begin{array}{l}\text { Attention } \\
\text { deficit }\end{array}$ & \begin{tabular}{|l} 
girls \\
boys
\end{tabular} & $\begin{array}{l}6 \\
6\end{array}$ & $\begin{array}{l}17 \\
15 \\
\end{array}$ & $\begin{array}{l}0 / 00 \\
0.00\end{array}$ & $\begin{array}{l}1 / 00 \\
1.00 \\
\end{array}$ & $\begin{array}{l}1 / 00 \\
1.00 \\
\end{array}$ & $\begin{array}{l}0 / 00 \\
0.00\end{array}$ \\
\hline \begin{tabular}{|l|}
$\begin{array}{l}\text { Non-Verbal } \\
\text { communication }\end{array}$ \\
\end{tabular} & \begin{tabular}{|l|} 
girls \\
boys
\end{tabular} & $\begin{array}{l}2 \\
2 \\
\end{array}$ & $\begin{array}{l}17 \\
15 \\
\end{array}$ & $\begin{array}{l}0 / 013 \\
0.014\end{array}$ & $\begin{array}{l}0 / 00 \\
0.00\end{array}$ & $\begin{array}{l}0 / 00 \\
0.08\end{array}$ & $\begin{array}{l}0 / 08 \\
0.00\end{array}$ \\
\hline $\begin{array}{l}\text { Verbal } \\
\text { communication }\end{array}$ & \begin{tabular}{|l|} 
girls \\
boys
\end{tabular} & $\begin{array}{l}5 \\
4\end{array}$ & $\begin{array}{l}17 \\
15 \\
\end{array}$ & $\begin{array}{l}0 / 00 \\
0.00\end{array}$ & $\begin{array}{l}0 / 00 \\
0.00\end{array}$ & $\begin{array}{l}0 / 00 \\
0.00\end{array}$ & $\begin{array}{l}0 / 00 \\
1.01 \\
\end{array}$ \\
\hline \begin{tabular}{|l|}
$\begin{array}{l}\text { General } \\
\text { colloquial skills }\end{array}$ \\
\end{tabular} & \begin{tabular}{|l|} 
girls \\
boys
\end{tabular} & $\begin{array}{l}7 \\
5\end{array}$ & $\begin{array}{l}17 \\
15 \\
\end{array}$ & $\begin{array}{l}0 / 00 \\
0.02\end{array}$ & $\begin{array}{l}0 / 00 \\
0.00\end{array}$ & $\begin{array}{l}0 / 00 \\
1.00\end{array}$ & $\begin{array}{l}0 / 00 \\
0.00\end{array}$ \\
\hline \begin{tabular}{|l|} 
Subject \\
maintaining
\end{tabular} & \begin{tabular}{|l|} 
girls \\
boys
\end{tabular} & $\begin{array}{l}4 \\
3\end{array}$ & $\begin{array}{l}17 \\
15 \\
\end{array}$ & $\begin{array}{l}0 / 00 \\
0.02\end{array}$ & $\begin{array}{l}0 / 00 \\
0.00\end{array}$ & $\begin{array}{l}1 / 10 \\
0.00\end{array}$ & $\begin{array}{l}0 / 00 \\
0.00\end{array}$ \\
\hline \begin{tabular}{|l|} 
Basic social \\
language skills
\end{tabular} & \begin{tabular}{|l|} 
girls \\
boys
\end{tabular} & $\begin{array}{l}7 \\
5\end{array}$ & $\begin{array}{l}17 \\
15\end{array}$ & $\begin{array}{l}0 / 00 \\
0.00\end{array}$ & $\begin{array}{l}0 / 00 \\
0.00\end{array}$ & $\begin{array}{l}1 / 00 \\
0.00\end{array}$ & $\begin{array}{l}0 / 00 \\
0.00\end{array}$ \\
\hline
\end{tabular}

\subsection{RELATIONSHIP BETWEEN GENDER AND COMMUNICATION ABILITY FACTORS (LANGUAGE APPLICATION SKILLS)}

One-way ONOVA analysis also revealed a significant differences between the age of children with ADHD for non-verbal communication skills (mean values7/029, $\mathrm{t}(34)=30$ ), verbal communication (mean value7/00, $\mathrm{t}(34)=32$ ) and general colloquial skills (mean value $7 / 00, \mathrm{t}(34)=31$ ). Similar age differences have been observed in subject maintaining factors (mean value 7/00, t (34) =30) and basic social language skills (mean value $7 / 00, \mathrm{t}(34)=32$ ) (data shown in table 9).

Table 9. Results of gamma test for the relationship between age and the rate of communication skills problems in children with attention deficit/hyperactivity disorder $(\mathbf{N}=34)$

\begin{tabular}{|l|l|l|l|l|}
\cline { 2 - 5 } \multicolumn{1}{c|}{} & $\begin{array}{l}\text { Gamma } \\
\text { value }\end{array}$ & $\begin{array}{l}\text { Asymptotic } \\
\text { standard error }\end{array}$ & $\begin{array}{l}\text { Approximate } \\
\text { t score }\end{array}$ & $\begin{array}{l}\text { Approximate } \\
\text { significance }\end{array}$ \\
\hline Age/Non-Verbal communication skills & -0.175 & 0.424 & 0.427 & 0.669 \\
\hline Age/Verbal communication skills & 0.143 & 0.349 & 0.405 & 0.685 \\
\hline Age/General colloquial skills & -0.016 & 0.360 & -0.405 & 0.964 \\
\hline Age/Subject maintaining skills & -0.458 & 0.284 & -1.525 & 0.127 \\
\hline Age/Basic social language skills & -0.095 & 0.326 & -0.289 & 0.772 \\
\hline
\end{tabular}

* We do not assume an empty hypothesis.

** Using criteria and assuming an empty hypothesis. 


\section{DISCUSSION AND CONCLUSION}

Language facilitates the behavior's organizing and planning. It also enables a person to anticipate opportunities for thinking in past behavior and as a result, prevents undesirable behaviors. Language ability is important for the development of internal speech or "self-talk", as it is found important for behavioral control as well as communication in the social domain (Barkly, 1997). Accordingly, language can be considered as a means of cognition, and thus can play an important role in interventions. This is proved by the research finding, revealing the non-verbal communication skills as the most problematic area in the three areas of the pragmatic skills, as shown in table 1, that the majority of subjects $(\mathrm{N}=26)$ have severe problems with their Non-verbal skills; that this might be because of much more cognitive impairments in these children.

Regarding the existence of relationship between pragmatic deficits and children with ADHD, the results of this study is in line with Bruce et al. (2005) and Staikuva et al. (2013 conducted on cognitive impairment and children with ADHD. Also, the results of this study should not be considered as a preliminary one because the study group is selected from a social context and is representative of a population of ADHD. Although previous studies have examined children with increased levels of attention deficit/hyperactivity disorder (ADHD) (e.g. Bignell \& Cain, 2007; Leonard et al., 2011), our sample was carefully diagnosed based on a comprehensive assessment of ADHD.

The results of the analysis also showed that most of the problems related to pragmatic skills are occurring in earlier ages, and as the age increases, these problems decrease. And, in accordance with data from tables $7 \mathrm{a}, \mathrm{b}$ boys had more problems in both subject maintaining skills $(\mathrm{p}=0 / 02)$ and in nonverbal communication ( $\mathrm{p}=0 / 014)$ compared to girls. These gender differences are, to some extent, in contrary to the results of Trillingsgaard et al. (2014), as contrary to the differences in the rate of referral of boys (47\%) and girls (53\%) to the medical and counseling center, it seems that boys in clinical samples, especially in non-verbal skills and their general conversations, were more problematic than those of girls. This while Trillinsgard et al. (2014) showed that girls have more problems with social and cognitive skills.

As a result of the research, it can be stated that the evaluation of the language use has always posed several challenges. First, language is a heterogeneous construction that involves a sequence of verbal and nonverbal skills and makes it difficult to evaluate using a single scale (Landa, 2005; Russell, 2007). In addition, as pragmatics is closely related to social context by definition, then assessing pragmatic skills through a standardized test may not reflect aa individual's actual ability to use language in a natural situation. As a result, a combination of measurements were used in the present study. In general, ANOVA analyzes showed that after controlling scores related to 
communicative skills, children with attention deficit/hyperactivity disorder had significantly weaker language skills.

The accuracy of this claim is better understood according to the results obtained in this study, as these results showed a significant relationship between ADHD disorder and the main factors of communication ability, i.e non-verbal skills, verbal skills and general conversational skills. The results of the present study were in complete agreement with the results of Bruce et al. (2006) and Staikova et al. (2013) on the relationship between pragmatic impairments and ADHD symptoms. Therefore, it can be concluded that there is a close relationship between pragmatic skills and the central symptoms of Attention Deficit/Hyperactivity Disorder in children.

\section{REFERENCES}

Abdollahian, Ebrahim, Shakerri, \& Vosoogh 2004. Investigation of ADHD prevalence in children of elementary school ages at Mashhad, in academic year 2001-2002. Medical Journal of Mashhad, Medical Sciences University, No. 85, year 47th, autumn 2003, pp 275-280. (Persian)

American Psychology Association. (1994). Diagnostic and statistical manual of mental disorders, 4th Edn. Washington, DC: Author.

Baird, J, Stevenson, JC. \& Williams, DC. (2000). The evolution of ADHD: A disorder of communication? The Quarterly Review of Biology, 75, 17-35.

Barkly, RA. (1997). ADHD and the Nature of Self-Control. The Guilford Press, New York.

Barkly, RA. 2002. Attention-Deficit/Hyperactivity Disorder. Child Psychology. Pp 276-320.

Biederman, J, Stephen, F., \& Millberger, S. (1996). Predictors of persistence and remission of ADHD into adolescence: results from a four-year prospective follow-up study. J Am Acad Child Adolesc Psychiatry 35:343-351, 1996b.

Bingel, S., \& Cain, K. (2007). Pragmatic aspects of communication, language, and comprehension in groups of children differentiated by teacher ratings of inattention hyperactivity. British Journal of Developmental Psychology, 25, 499-512.

Bishop, D., Chan, J., Adams, C, Hartley, J. \& Weir, F. (2000). Evidence of disproportionate pragmatic difficulties in a subset of children with specific language impairment in children. Journal of Speech, Language and Hearing Research, 42(6), 1295-1310.

Bishop, D.V., \& Baird, G. (2001). Parent and teacher report of pragmatic aspects of communication: Use of the children's communication checklist in a clinical setting. Developmental Medicine and Child Neurology, 43, 809-818.

Bruce, B., Therlind, G., \& Nettlbeadt, U. (2006). ADHD and language impairment: A study of the parent questionnaire FTF (Five to Fifteen). European Child and Adolescent Psychiatry, 15, 52-60. 
Camarata, S.M., \& Gibson, T. (1999). Pragmatic language deficits in attention-deficit hyperactivity disorder (ADHD). Mental retardation and developmental disabilities research reviews, 5, 207-214.

Cohen, NJ, Davine, M., Horodezky, N., Lipsset, L, \& Issacson, L. 1993. Unsuspected impairment in psychiatry disturbed children prevalence, language, and behavioral characteristics. Journal of the American Academy of Child and Adolescent Psychiatry, 32, 595-603.

DuPaul, G.J., Power, T.J., Anastopoulos, A.D., \& Reid, R. (1998). ADHD rating scale- $I V$. New York: Guildford.

Ellis, R. (1999). Learning a second language through interaction. Amsterdam/ Philadelphia: John Benjamins. Pp. 238-239.

Fischer, M, Barkly, RA. \& Smallish, L. (2002). Young adult follow up of hyperactive children: self-reported psychiatric disorders, comorbidity, and the role of children conduct problems and teen CD. J Abnorm Child Pstchol 300:463-475.

Friedman, S.R., Rapport, L.J., Lumley, M., Tzelepis, A., VanVoorhis, A., \& Stettner, L., et al. (2003). Aspects of social and emotional competence in adult attention deficit/hyperactivity disorder. Neuropsychology, 17, 50-58.

Gallagher, T. (1991). Language and social skills: Implications for clinical assessment and intervention with school-age children. In T. Gallagher (Ed.), Pragmatics of language: Clinical practice issues. San Diego, CA: Singular Publishing Group. Pp. 11-41.

Geurts, H.M., \& Embrechts, M. (2008). Language profiles in ASD, SLI, and ADHD. Journal of Autism and Developmental Disorders, 38, 1931-1943.

Geurts, H.M., Verte, S., Oosterlaan, J., Roeyers, H., Hartman, C.A., Mulder, E.J., , . \& Sergeant, J.A. (2004). Can the children's communication checklist differentiate between children with autism, children with ADHD, and normal controls? Journal of Child Psychology and Psychiatry, 45, 1437-1453.

Hodgens, J.B., Cole, J., \& Boldizar, J. (2000). Peer-based differences among boys with ADHD. Journal of Clinical Child Psychology, 29, 443-452 .

Humphries, T., Koltun, H., Malone, M., \& Roberts, W. (1994). Teacheridentified oral language difficulties among boys with attention problems. Journal of Developmental and Behavioral Pediatrics, 15, 92-98.

Kadesjo, B, Janols, LO., Korkman, M., Mickelsson, K., Strand, G., Trillinsgaard, A. \& Gillberg, C. (2004). The FTF (Five to Fifteen): the development of a parent questionnaire for the assessment of ADHD and comorbid conditions. Eur Child Adolesc Psychiatry, 13 (3), 3-13.

Landa, RJ. (2005). Assessment of social communication skills in preschoolers. Mental Retardation and Developmental Disabilities Research Reviews, 11, 247-252.

Leonard, MA., Milich, R., \& Lorch, EP. (2011). The role of pragmatic language use in mediating the relation between hyperactivity and inattention and social skills problems. Journal of Speech, Language, and Hearing Research, $54,567-579$. 
Mc Tear, MF., \& Conti-Ramsden, G. (1992). Pragmatic disability in children. London: Whurr Publishers, Pp. ix+235.

Mikami, A.Y., Ransone, M.L., \& Calhoun, C.D. (2011). Influence of anxiety on the social functioning of children with and without ADHD. Journal of Attention Disorders, 15, 473-484.

Prutting, CA. (1982). Pragmatics as social competence. Journal of Speech and Hearing Disorders, 47, 123-134.

Russell, RL. (2007). Social communication impairments: Pragmatics. Pediatric Clinics of North America, 54, 483-506.

Shahim, S, Mehrangiz, L., \& Yousefi, F. 2007. Prevalence of attention deficit/ hyperactivity disorder in preschool children. Iranian Journal of Pediatric Diseases, 17 (2), 211-216. (Persian)

Staikova, E., Gomes, H., Tartter, V., Mc Cabe, A., \& Halperin, JM. (2013). Pragmatic deficits and social impairment in children with ADHD. Journal of Child Psychology and Psychiatry, 54(12), 1275-1283. doi:10.1111/jcpp.12082

Tannock, R, \& Brown, TE. (2000). Attention deficit disorders with learning disorders in children and adolescents. In T. E. Brown (Ed.), Attention deficit disorders and comorbidities in children, adolescents, and adults, Washington, DC: American Psychiatric Press.

Trillingsgaard, A, Dammn, D.., Sommer, S., Jepsen, J., Richardt, M., Østergaard, O., Frydenberg, M., \& Thomsen, PH. (2004). Developmental profiles on the basis of the FTF (Five to Fifteen) questionnaire. European Child and Adolescent Psychiatry 13:3:iii39-iii49.

Turksra, LS, Mc Donald, S., \& Kaufman, PM. (1995). Assessment of pragmatic communication skills in adolescents after traumatic brain injury, 10, $329-345$.

Von Briesen, P.D. (2007). Pragmatic language skills of adolescents with $A D H D$. Unpublished dissertation submitted to the faculty of the graduate school, Marquette University.

Weiss, G, \& Hechman,. LT (1993). Hyperactive children grown up: ADHD in children, adolescents and adults. Second Edition. New York, Guilford, 1993.

Whalen, CK, \& Hemker, B. (1991b). Therapies for hyperactive children: Comparisons, combinations, and compromises. Journal of Consulting and Clinical Psychology, 59, 126-137.

Winsler, Diaz, Mc Carty, Atencio, \& Chabay. (2000). Verbal self-regulation overtime in Preschool children at risk for attention and behavior problems. Journal of child psychiatry, 41(7), 855-886.

Zare Abadi, M.ehdi, \& Najafi, K.amran (2014). Prevalence of attention deficit/ hyperactivity disorder and comorbidity with learning disorder in elementary school students. Journal of Learning Disabilities, 3(4), 25-43. (Persian) 